\title{
Editorial
}

\section{Modeling Transparency in Roles: Moving from Authorship to Contributorship}

\section{Sarah Fitzgerald, John Budd, Penny Beile, and Wendi Kaspar}

The Association of College and Research Libraries' Framework for Information Literacy describes several principles for appropriate use of information, including "Information has value." "Information has value" in operation means giving credit to the original ideas of authors and recognizing "the skills, time, and effort needed to produce knowledge." College $\mathcal{E}$ Research Libraries wants to align itself with this principle by creating transparency around which authors contribute what valuable roles to its publications. Given the increasing number of coauthored articles in scholarly publishing, teasing apart who is responsible for what is a growing task in the evaluation of scholarly work. The journal, its editors, and the editorial board are making a concerted effort to model these values, while still maintaining high standards and impartiality. We are also seeking to evolve the journal and be responsive to the climate around ethics, bias, and openness - and the editorial board members have been heavily engaged in this effort. Hand in hand with this priority to bring transparency to the reviewer process and decision making, there has also been discussion about how to advocate for more transparency in author roles and assigning credit. From my perspective as editor, more clarity and openness around the contributors to paper submissions may provide a better understanding of the project described, but also a more accurate picture of how individuals contributed. Having a model that is more inclusive about contributor roles and the expectations of authorship would also minimize the questions or issues that periodically arise with submissions. I have discussed some of these issues and the potential solutions in previous editorials. ${ }^{1}$ Adoption of author role guidelines can only encourage more thoughtful discussion with authors that will reduce the possibility of confusion or conflict.

Authorship, contributorship, and acknowledgements are more controversial than might be assumed. In higher education, authorship has long been the currency for promotion and has prompted issues from author disputes to accusations of research misconduct. Authorship seems very finite - and in some views, plays to the elitism and marginalization that has become a widespread complaint in academia.

Following the ongoing discussion that the Board has had about transparency and authorship, there was recently a decision to jump into the author role issue more actively. The CRediT taxonomy ${ }^{2}$ has been at the center of many conversations (including a high-level recognition from $\mathrm{NISO}^{3}$ ) and was used as the jumpoff point for this effort. The Consortia Advancing

(C2020 Sarah Fitzgerald, John Budd, Penny Beile, and Wendi Kaspar, Attribution-NonCommercial (https://creativecommons.org/licenses/by-nc/4.0/) CC BY-NC. 


\section{Modeling Transparency in Roles: Moving from Authorship to Contributorship}

Standards in Research Administration Information has developed a taxonomy for assigning credit to authors in science publications named CRediT (Contributor Roles Taxonomy). This taxonomy consists of 14 contributions that authors can report having executed for a given publication. These are Conceptualization, Data Curation, Formal Analysis, Funding Acquisition, Investigation, Methodology, Project Administration, Resources, Software, Supervision, Validation, Visualization, Writing (Original Draft), and Writing (Review \& Editing). These roles were developed for scholars in the sciences; as such, they are not perfectly reflective of the workflows and values of social science scholars who publish in College $\mathcal{E}$ Research Libraries, so a task force was formed to adapt the taxonomy to better represent the principles of social science publishing.

After some discussion with the board, Sarah Fitzgerald offered to be part of a task force-joined by John Budd and Penny Beile - to investigate and make a recommendation about an author roles framework. They had extensive conversation via email and, I have to say, it was an impressive exchange-with each person offering up expertise and evidence, analyzing different aspects to provide a more fulsome understanding of the issue and of the environment around the journal and its authors. What was more amazing is the speed with which it happened - from formation of the group to final recommendation to the Board at large lasted 2 weeks!

There were a lot of exchanges and I feel that it would be useful for readers to see some of it-not just to understand how the planning for the journal works, but also what a high performing team, working asynchronously online, looks like (an especially useful model in this particular time).

The task force analyzed articles published in CERL in 2019 in an effort to analyze the types of articles, methodologies, framing, and so on. Of the 49 articles published in 2019, 47 had some element of a research methodology. Two articles (Jan 2019 Hosier and July 2019 Folk) were "more conceptual, 'think' pieces." It was inferred that roles on the taxonomy that could apply to these two pieces are literature synthesis, writing, and editing. Conceptualization, as currently defined, would not apply as well as any fields/roles that have to do with data, but that was not a major concern as these pieces are generally single authored and, thus, the attribution of credit is less in doubt. Overall, with the small number of articles like this, this was not considered a primary concern for the task force.

While examining the article types and implicit roles of articles in 2019, the task force also assessed the acknowledgements included in any articles. Acknowledgements are one of the contributor roles that are often ambiguous. COPE offers guidelines around authorship and contributorship, asserting that "Clear policies (that allow for transparency around who contributed to the work and in what capacity) should be in place for requirements for authorship and contributorship as well as processes for managing potential disputes." ${ }^{4}$ They tended to fall into a few major categories:

- Reviewing, editing, feedback (for survey instruments, pretest, draft manuscripts): 17

- Data collection/coding/access to data or sharing: 10

- Scholarship/funding: 8

- Mentor/consulting/general assistance: 7

- Survey respondents/participants: 4

- Data analysis assistance/presentation: 4

- Assistance with an earlier study: 2 
The acknowledgements were considered significant, as there are times when such a contributor's role may rise to the level of authorship, particularly if the project or paper could not move forward without their participation. One comment from a task force member (Sarah) expressed this sentiment with: "I hope that based on our taxonomy more library science authors will include their data analysts and data visualizers as authors rather than merely acknowledging them. This is important work which requires informed decision making and makes enormous differences in the findings and conclusions of articles."

Considering specific roles, the task force noted that there would be some frequently appearing roles: literature synthesis, conceptualization, methodology, instruments, investigation, formal analysis, interpretation, visualization, writing, and editing. Three roles-discussed early in the project-prompted some longer consideration as their roles in library research are evolving:

Project Management: There were few articles, if any, that had the scope or involvement to warrant using this role. The discussion of this role indicated that a project manager may contribute to a project in some way that justifies authorship (such as conceptualization or data collection), but the act of project management, in and of itself, does not constitute authorship. The task force went on to suggest that project management contributions be noted in the acknowledgements section since more of an acknowledgement-worthy contribution since it does not shape the findings or conclusions.

Data Curation: Only one article mentioned a data set and included a link to the data in the acknowledgements section. Although data curation may not be mentioned by articles, it is a contribution that requires expertise and shapes the findings and conclusions, which seems like a significant piece of authorship for any quantitative article and some qualitative articles, even though it is fairly invisible in many published versions. The task force recommended that the data curation role be retained to encourage more authors to provide data sets and/ or curation practices.

Software Development: Only one article mentioned development of a Python API for citation analysis (Jan 2019 White), indicating that there was not a pressing need for this role at this time. However, there was recognition that the role of software in library research may be growing and that it was worth including this role to future-proof the taxonomy.

The task force felt many of the roles identified by CRediT were also of great importance to social science authors. Conceptualization of research goals, development of methodology, investigation through data collection, data curation, data analysis, data visualization, writing, and editing are greatly important across disciplines. At first, we were not sure that software development was important enough to library science authors to include in our taxonomy; but, after discussion, we decided this role will have growing importance in our field, and it was important for our taxonomy to encompass the future of our field as well as the present.

The task force felt it was imperative to add several categories to reflect processes valued by social scientists, which did not appear in the existing taxonomy. We felt it was vital to include a category about Literature Synthesis. Reviewing and analyzing existing scholarly knowledge regarding the research question(s) is very important to social scientists, to identify the limitations of previous research and build a case for the need for the current study. As members of a field focused on providing access to knowledge, librarians value this work highly. Interpretation of the results was also a contributor role we strongly felt needed to be added. Because library science is an applied field, rather than an abstract discipline, interpretation 
of the results for practitioners is central to research in the field. This is true of many other applied social sciences as well. The other category we felt needed to be added was instrumentation. This need did not stand out to us instantly like the other two. It emerged from a review of published articles in the journal during the last year. We noticed the appearance of many survey instruments and interview protocols either included as appendices or alluded to by the articles. We felt this work was significant and deserved acknowledgement.

There are five fields from the CRediT taxonomy that the task force did not recommend including in an adapted taxonomy for social science authors. The task force was guided in this by the recommendations of the Council of Science Editors, who state that "contributions that alone do not justify authorship include: assisting the research by providing advice, providing research space, departmental oversight, obtaining financial support, isolated analyses, or providing reagents/patients/animals/other study materials. ${ }^{\prime 5}$ This led us to eliminate Funding Acquisition, Project Administration, Resources, Supervision, and Validation from our list of author contribution roles. While all of these roles are significant contributions to research, they do not constitute authorship in themselves. We recognize that many individuals who contribute in these ways to research also contribute to articles in ways that do merit authorship credit and should be listed as authors for their work in those areas. We encourage authors to honor contributions falling into these five categories as acknowledgements.

The plan is to roll out this model during the 2021 volume year. There will be some additional guidance added to the journal site and the attendant libguide as well as mentoring authors of accepted papers through the adoption of this model.

\section{Library Science Author Roles}

The purpose of identifying responsibility for the following roles is to acknowledge intellectual contributions, establish accountability, and increase transparency in library science research.

You are encouraged to identify all authors who have contributed to these roles. Multiple contributors to any one field also may be identified by the proportional effort expended: lead, equal, or support.

Individuals who contributed, but not to the point of warranting authorship, should be recognized in Acknowledgements. This might include those who reviewed or commented on study processes or manuscript drafts, provided access to data or assisted with data collection or analysis, served in an oversight or advisory capacity, facilitated access to instrumentation or research populations, or other similar functions.

Literature Synthesis: Reviewing and analyzing existing related scholarly knowledge regarding the research question(s). Identifying limitations of prior research and need for current study.

Conceptualization: Formulation of research goals or hypotheses.

Methodology: Development of methodology; creation of models.

Instruments: Development of original research instruments such as surveys or interview protocols.

Software: Development of software for data collection or analysis.

Investigation: Collection and coordination of data/evidence. This can include activities such as survey administration, interviewing participants, web scraping, or administering learning assessments.

Data Curation: Data management activities for initial use and later reuse. For example, 
data cleaning, documenting data and workflow, and maintenance.

Data Analysis: Conducting statistical techniques, thematic analysis, or other formal techniques to analyze study data. This may include use of software such as statistical packages or qualitative analysis software.

Interpretation: Analysis of the results for application to practice or future research.

Visualization: Creation of data visualization, including tables, charts, figures, and graphs. Writing: Creation of the published work (including substantive translation).

Editing: Critical review, commentary, or revision, including pre- or post-publication stages.

\section{Notes}

1. Wendi Arant Kaspar, "Authorship Norms: Credit and Roles," College \& Research Libraries 79, no. 2 (2018), https://crl.acrl.org/index.php/crl/article/view/16910/18573; Bruce E. Herbert and Wendi Arant Kaspar, "Authorship and the Consideration of Alternatives," College $\mathcal{E}$ Research Libraries 80, no. 1 (2019), https://crl.acrl.org/index.php/ crl/article/view/17542/19377.

2. Contributor Roles Taxonomy website, https://casrai.org/credit/.

3. Find out more about CRediT by visiting http://credit.niso.org/.

4. COPE website, https://publicationethics.org/authorship.

5. "Authorship and Authorship Responsibilities," Council of Science Editors website, https://www.councilscienceeditors.org/resource-library/editorial-policies/white-paper-on-publication-ethics/2-2-authorship-andauthorship-responsibilities/. 